Discussion Paper \#2002-2

\title{
RATIONALITY ON FINAL DECISIONS LEADS TO SEQUENTIAL EQUILIBRIUM
}

by

Ryuichiro ISHIKAWA and Takashi MATSUHISA

April, 2002 


\title{
RATIONALITY ON FINAL DECISIONS LEADS TO SEQUENTIAL EQUILIBRIUM*
}

\author{
RYUICHIRO ISHIKAWA \\ Graduate School of Economics, Hitotsubashi University \\ Naka 2-1, Kunitachi-shi, Tokyo 186-8601, Japan \\ E-mail: MXB02762@nifty.ne.jp \\ TAKASHI MATSUHISA ${ }^{\dagger}$ \\ Department of Liberal Arts and Sciences, Ibaraki National College of Technology \\ 866 Nakane, Hitachinaka-shi, Ibaraki 312-8508, Japan \\ E-mail: mathisa@ge.ibaraki-ct.ac.jp
}

\begin{abstract}
The purpose of this article is to investigate epistemic conditions for a sequential equilibrium in an extensive form game with imperfect information: If players mutually know that all players maximize their own expected payoffs at any information sets in their final decisions then their behaviors with belief yield a sequential equilibrium. This result is an extension of Aumann (1995, Games and Economic Behavior, 8:6-19) in a perfect information game. In this article, we propose the notion of $\mu$-rationality, by which we mean that player knows that he maximizes his own payoff according to the common-belief $\mu$. Furthermore we introduce the notion of $\mu$-consistency in imperfect information game. Our main theorem states that mutual knowledge of both $\mu$-rationality and $\mu$-consistency induces a sequential equilibrium outcome in an extensive form game.
\end{abstract}

Keywords: Knowledge, Rationality, Epistemic conditions, Backward induction, Sequential equilibrium.

\section{Introduction}

This paper investigates what epistemic conditions induce a sequential equilibrium, that is, what each player should know in order to achieve the sequential equilibrium in a given game. There are many equilibrium solutions in an extensive form game, however it is not clear how players achieve these solutions. This paper aims to fill this gap for sequential equilibrium in an extensive form game with imperfect information.

In a normal form game, Aumann and Brandenburger (1995) gives epistemic

\footnotetext{
*The early version "Knowledge, Rationality, and Sequential Equilibria" was presented at the Economic Theory Workshop of Hitotsubashi University in July 2001 and at the annual meeting of the Japanese Economic Association, Hitotsubashi University in October 2001. An abstract of this paper was presented in the R.I.M.S. Symposium of 'Mathematical Economics,' Kyoto University, Kyoto (Japan), December 7-9, 2001.

${ }^{\dagger}$ Partially supported by the Grand-in-Aid for Scientific Research(C)(2)(No.12640145) in the Japan Society for the Promotion of Sciences.
} 
conditions for leading to Nash equilibrium: Suppose that the players have a common prior, that their payoff functions and their rationality are mutually known, and that their conjectures for the opponents' actions are commonly known. Then the conjectures form Nash equilibrium.

In an extensive form game there is a contradictory relationship between players' rationality and solution concepts. The contradiction is informally presented by Rosenthal (1981) and is formally investigated by Reny (1992) and Ben-Porath (1997). They show that players' rationality at the root in the extensive form game does not always lead to the backward induction outcome by examining the centipede game.

On the other hand Aumann (1995) establishes the theorem that players' rationality at every node in a perfect information game can lead to the backward induction outcome.

In this paper we investigate in the same line of Aumann. We extend his result into in an imperfect information game as follows:

Main Theorem. The mutual knowledge of $\mu$-rationality in players' final decisions leads to a sequential equilibrium of an extensive form game with imperfect information.

Precisely, if everybody knows that each maximizes his own expected payoff according to the common belief $\mu$ at each information set in their final decisions, then the assignment associated with $\mu$ induces a sequential equilibrium.

This paper is organized as follows: In Section 2 we recall an extensive form game and the sequential equilibrium based on Kreps and Wilson (1982). In addition, we introduce knowledge of players and $\mu$-rationality. In section 3 we formally state the main theorem and give examples to illustrate it. In section 4 we give the proof of the main theorem, and in the final section we conclude some remarks.

\section{Game and Knowledge}

\subsection{Extensive form Games}

We consider a finite extensive form game. By this we mean a structure $G=\langle(T, \prec$ 
), $\left.N,\left(\mathcal{I}_{i}\right)_{i \in N},\left(A_{i}\right)_{i \in N},\left(u_{i}\right)_{i \in N}\right\rangle$ consisting of as follows: $T$ is the finite set of nodes that is divided into the set of players' decision nodes $X$ and the set of the terminal nodes $Z$. We assume there is no chance moves for simplicity. ${ }^{\mathrm{a}}(T, \prec)$ forms a tree with the unique root: The relation $\prec$ is a totally order on the predecessors $P(x)$ of each member $x$ in $T$ and $p(x)$ is the immediate predecessor of $x . N$ is a set of finitely many players. For each $i \in N, X_{i}$ is the subset of $X$ that consists of $i$ 's decision nodes and thus $X$ is the disjoint union of all the sets of $X_{i}$ for $i \in N$. We denote by $\iota(x)$ the player making his decision at $x \in X$.

The information that player $i$ possesses is represented by $i$ 's information partition $\mathcal{I}_{i}$ on $X_{i}$ consisting of components $I_{i}$ called $i$ 's information set. When a set $I_{i} \in \mathcal{I}_{i}$ contains a node $x \in X_{i}$, we denote it by $I_{i}(x)$ (or simply by $I(x)$.) Each information set is identified with the set of all the decision nodes among which the player can not distinguish. In addition $\mathcal{I}$ denotes the disjoint union of all $\mathcal{I}_{i}$ for $i \in N$.

Each player $i$ has a feasible action set $A_{i}(I)$ at every $I \in \mathcal{I}_{i}$. Since each of $i$ 's information sets is the set of nodes that she can not distinguish, the feasible action sets $A_{i}(x), A_{i}\left(x^{\prime}\right)$ at $x, x^{\prime} \in I$ are identified with each other, which denotes $A_{i}(I)$. We denote by $A_{i}$ the set of all profiles of $i$ 's feasible-actions; that is, $A_{i} \equiv$ $\times_{I \in \mathcal{I}_{i}} A_{i}(I)$.

In this paper we focus on games with perfect recall. ${ }^{\mathrm{b}}$ An extensive form game $G$ is said to be with perfect recall if the following conditions are satisfied:

- For any two nodes in a same information set, it is impossible that one node is the predecessor of the other one.

- For any three nodes $x, x^{\prime}, x^{\prime \prime} \in X_{i}$ with $x^{\prime} \in I\left(x^{\prime \prime}\right)$ and $x \in P\left(x^{\prime}\right)$, there exist $\hat{x} \in I(x) \cap P\left(x^{\prime \prime}\right)$ and $a \in A_{i}(I(x))$ such that if $a$ respectively reaches $x^{\prime}$ and $x^{\prime \prime}$ then it is played at both $x$ and $\hat{x}$.

The assumption of perfect recall plays a crucial role in the main theorem. Let $u_{i}: Z \rightarrow \mathbf{R}$ be $i$ 's payoff function on the terminal nodes of $T$.

${ }^{a}$ We restrict our attention into the case that the number of the initial node is just one for simplicity. ${ }^{b}$ Kuhn (1953). 
A local strategy at $I \in \mathcal{I}_{i}$ for player $i$ is a probability distribution $b_{i}^{I}$ on $A_{i}(I)$, and $i$ 's behavior strategy $b_{i}$ is the profile $\left(b_{i}^{I}\right)_{I \in \mathcal{I}_{i}}$. A behavior strategy $b_{i}$ is called $i$ 's pure strategy if each component of $b_{i}$ assigns the probability one to the specific action of $A_{i}(I)$ at each information set $I$. Let $\mathcal{B}_{i}(I)$ denote the set of all local strategies at $I$ and $\mathcal{B}_{i}$ the set of all behavior strategies for player $i$. We set $\mathcal{B}=\times_{i \in N} \mathcal{B}_{i}$ the set of all profiles of behavior strategies.

Each strategy $b \in \mathcal{B}$ induces the probability distribution $\boldsymbol{P}^{b}$ on $T$ defined as follows: For $x \in T$,

$$
\boldsymbol{P}^{b}(x):=\prod_{a \in \pi(x)} b(a)
$$

where $\pi(x)$ is the set of all actions reaching $x$ from the root. The formula (2.1) represents the probability to reach $x$ from the root calculated by the strategies on $P(x)$. i's expected utility $U_{i}$ induced from $\boldsymbol{P}$ on $\mathcal{B}$ is defined by

$$
U_{i}(b):=\sum_{z \in Z} \boldsymbol{P}^{b}(z) u_{i}(z)
$$

By Kuhn's theorem in Kuhn (1953) we restrict our attention to behavior strategies; hereafter behavior strategies are simply called strategies in this paper.

\subsection{Sequential Equilibrium ${ }^{\mathrm{c}}$}

A system of beliefs is the class of probability distributions $\mu$ on each information set $I \in \mathcal{I}$; hence $\sum_{x \in I} \mu(x)=1$ for each $I \in \mathcal{I}$. Let $\mu(x)$ interpret as a belief assigned by $\iota(x)$ to $x \in I$ if an information set $I$ is reached. Let $\mathcal{M}$ denote the set of beliefs. Each member of $\mathcal{B} \times \mathcal{M}$ is called an assessment. Given an assessment $(b, \mu) \in \mathcal{B} \times \mathcal{M}$, we define the conditional probability $\boldsymbol{P}^{b, \mu}(\cdot \mid I)$ over $Z$ by

$$
\boldsymbol{P}^{b, \mu}(z \mid I)= \begin{cases}0 & \text { if } x \notin P(z) \cap I \\ \mu(x) \prod_{a \in \pi(x, z)} b(a) & \text { if } x \in P(z) \cap I,\end{cases}
$$

where $\pi(x, z)$ is the set of actions taken to reach $z$ from $x \in I$. This formula represents the probability of player's assessment of reaching each terminal node ${ }^{c}$ Kreps and Wilson (1982). 
when she is at an information set $I$. Then we define the conditional expectation $U_{i}^{\mu}$ under $i$ 's information set $I$ by

$$
U_{i}^{\mu}(b \mid I):=\sum_{z \in Z} \boldsymbol{P}^{b, \mu}(z \mid I) u_{i}(z)
$$

Let $\mathcal{B}^{+}$denote the set of strategies $b \in \mathcal{B}$ such that $b(a) \supsetneqq 0$ for any $a \in A$, and $\mathcal{M}^{+}$the subset of $\mathcal{M}$ which consists of $\mu \in \mathcal{M}$ such that $\mu(x) \supsetneqq 0$ at each $x \in X$. For given $b \in \mathcal{B}^{+}$, we say that the belief $\mu$ is associated with $b$ if it is defined by the Bayes' rule:

$$
\mu(x \mid b)=\boldsymbol{P}^{b}(x) / \sum_{\hat{x} \in I} \boldsymbol{P}^{b}(\hat{x}) .
$$

We can now define the sequential equilibria of $G$ as follows.

Definition 2.1. Let $G$ be an extensive form game. We denote by $\mathcal{S E}(G \mid I)$ the set of all the assessments $\left(b^{*}, \mu^{*}\right)$ satisfying both the conditions $\left(\mathbf{C}_{\mathbf{I}}\right)$ and $\left(\mathbf{S R}_{\mathbf{I}}\right)$ at an information set $I$ :

$\left(\mathbf{C}_{\mathbf{I}}\right)$ An assessment $\left(b^{*}, \mu^{*}\right)$ is consistent at the information set $I$. That is, there exists a sequence $\left\{\left(b^{n}, \mu\left(\cdot \mid b^{n}\right)\right)\right\} \subseteq \mathcal{B}^{+} \times \mathcal{M}^{+}$such that for all $x \in I$ and all $a \in A_{\iota(I)}(I)$,

$$
\lim _{n \rightarrow \infty}\left(b^{n}(a), \mu\left(x \mid b^{n}\right)\right)=\left(b^{*}(a), \mu^{*}(x)\right) .
$$

$\left(\mathbf{S R}_{\mathbf{I}}\right)$ An assessment $\left(b^{*}, \mu^{*}\right)$ is sequential rational at the information set I. That is, for the information set $I$ and for any alternative strategy profile $b_{i}^{\prime} \in \mathcal{B}_{i}$,

$$
U_{i}^{\mu}\left(b^{*} \mid I\right) \geqq U_{i}^{\mu}\left(b_{i}^{\prime}, b_{-i}^{*} \mid I\right),
$$

where $i=\iota(I)$ and $b_{-i}^{*}=\left(b_{j}^{*}\right)_{j \in N \backslash\{i\}}$.

Let $\mathcal{S E}(G)$ denote the intersection of $\mathcal{S E}(G \mid I)$ over $I \in \mathcal{I}$. We call $\left(b^{*}, \mu^{*}\right) \in \mathcal{S E}(G)$ a sequential equilibrium of the game $G$.

\subsection{Knowledge Structure on $G$}


Aumann (1995) introduces the partition model of knowledge on extensive form games. He shows that the backward induction outcome is reached by the common knowledge of rationality in perfect information games. We will extend the model of knowledge on perfect information games into that on imperfect information games.

A knowledge structure on an extensive form game $G$ is a quadruple

$$
\left\langle\Omega,\left(\Pi_{i}\right)_{i \in N},\left(K_{i}\right)_{i \in N}, \mathbf{b}\right\rangle
$$

consisting of the following structures and interpretations: $\Omega$ is a non-empty set, each element $\omega$ is called a state, a subset $E$ of $\Omega$ an event, and $\Pi_{i}$ is a mapping of $\Omega$ into $2^{\Omega}$ such that the image makes a partition on $\Omega$ consisting of components $\Pi_{i}(\omega)$ for $\omega \in \Omega$. To avoid confusion we call $\Pi_{i} i$ 's knowledge partition. Intuitively a component $\Pi_{i}(\omega)$ of $i$ 's knowledge partition is interpreted as the event consisting of all the states that player $i$ cannot distinguish from $\omega$. $i$ 's knowledge operator $K_{i}$ on $2^{\Omega}$ is defined by

$$
K_{i} E=\left\{\omega \in \Omega \mid \Pi_{i}(\omega) \subseteq E\right\} \quad \text { for } E \subseteq \Omega .
$$

A mapping $\mathbf{b}$ from $\Omega$ to $\mathcal{B}$ assigns a $|N|$-tuple $\left(\mathbf{b}_{i}(\omega)\right)_{i \in N}$ of players' strategies to each $\omega \in \Omega$.

We record the properties of the knowledge operator: ${ }^{\mathrm{d}}$ For any $E, F \subseteq \Omega$,

(N) $K_{i} \Omega=\Omega$

(M) If $E \subseteq F$, then $K_{i} E \subseteq K_{i} F$;

(K) $K_{i}(E \cap F)=K_{i} E \cap K_{i} F$;

(T) $K_{i} E \subseteq E$;

(4) $K_{i} E \subseteq K_{i}\left(K_{i} E\right)$;

(5) $\Omega \backslash K_{i} E \subseteq K_{i}\left(\Omega \backslash K_{i} E\right)$.

${ }^{d}$ Bacharach (1985). 
The mutual knowledge operator $K_{E}$ on $\Omega$ is defined by $K_{E} F=\bigcap_{i \in N} K_{i} F$. The event $K_{E} F$ is interpreted as that 'every player knows $F$.' An event $F$ is called publically known among the players if $F \subseteq K_{E} F$. The common-knowledge operator $K_{C}$ is defined by

$$
K_{C} E:=\bigcap_{k=1,2, \ldots\left\{i_{1}, i_{2}, \ldots, i_{k}\right\} \subseteq N} \bigcap_{i_{1}} K_{i_{2}} \cdots K_{i_{k}} E .
$$

The event $K_{C} E$ is interpreted as that 'all players know that all players know that - that all players knows $E$.'

Now, if $\phi$ is a function on $\Omega$ and $v$ is its value then $[\phi=v]$ (or simply $[v]$ ) denotes the event $\{\omega \in \Omega \mid \phi(\omega)=v\}$. Therefore for any $b_{i} \in \mathcal{B}_{i}$, we denote by $\left[b_{i}\right]$ the set $\left\{\omega \in \Omega \mid \mathbf{b}_{i}(\omega)=b_{i}\right\}$. We assume that every behavior strategy is publically known among the players; that is,

$$
\left[b_{i}\right] \subseteq K_{E}\left[b_{i}\right] \quad \text { for every } b_{i} \in \mathcal{B}_{i} .
$$

This is interpreted as that everybody knows every behavior strategy for each player. In view of the assumption (PK) we can observe that each strategies of player $i$ is $\Pi_{i}$-measurable, and thus $K_{i}\left[b_{i}\right]=\left[b_{i}\right]$ by $(\mathbf{T})$.

Example 1. Let $G$ be an extensive form game $\left\langle(T, \prec), N,\left(\mathcal{I}_{i}\right)_{i \in N},\left(A_{i}\right)_{i \in N},\left(u_{i}\right)_{i \in N}\right\rangle$. Let $\Omega=T \backslash Z$ and $\Pi_{i}$ the function from $\Omega$ to $2^{\Omega}$ defined by:

$$
\Pi_{i}(\omega)= \begin{cases}I_{i}(\omega) & \text { if } \omega \in X_{i} \\ \Omega \backslash X_{i} & \text { otherwise. }\end{cases}
$$

Let $\mathbf{b}_{i}^{I}: \Omega \rightarrow \mathcal{B}_{i}(I)$ be defined as follows: We let take $a \in A_{i}(I)$ and $b_{i} \in \mathcal{B}_{i}(I)$ for each $i \in N$, and we define

$$
\mathbf{b}_{i}^{I}(\omega)= \begin{cases}b_{i}(a) & \text { if } \omega \in I \in \mathcal{I}_{i} \\ 0 & \text { otherwise }\end{cases}
$$

We set $\mathbf{b}_{i}=\left(\mathbf{b}_{i}^{I}\right)_{I \in \mathcal{I}_{i}}$ as the profile of $\mathbf{b}_{i}^{I}$ over $i$ 's information sets, and set the mapping $\mathbf{b}$ on $\Omega$ into $\mathcal{B}$ by $\mathbf{b}=\left(\mathbf{b}_{i}\right)_{i \in N}$. Let us define the knowledge operator $K_{j}$ 
for player $j$ as follows:

$$
K_{j}\left[b_{i}^{I}\right]= \begin{cases}I & \text { if } i=j \\ \emptyset & \text { if } i \neq j,\end{cases}
$$

for any $b_{i}^{I} \in \mathcal{B}_{i}(I)$. Then for any $b_{i} \in \mathcal{B}_{i}$ and $b_{i}^{I} \in \mathcal{B}_{i}(I)$, it can be observed that $\left[b_{i}\right]=\sqcup_{I \in \mathcal{I}_{i}}\left[b_{i}^{I}\right] \subseteq \cup K_{E}\left[b_{i}^{I}\right]=\sqcup_{I \in \mathcal{I}_{i}} I$ by $(\mathbf{M})$, where the symbol $\sqcup$ denotes the disjoint union operator.

\subsection{Rationality and Consistency}

We introduce the notion of rationality which is an extension of rationality in Aumann (1995). Let $\mu$ be a system of beliefs. An player $i$ is said to be $\mu$-rational at $I \in \mathcal{I}_{i}$ if each strategy that $i$ does not know never yields his expected utility value according to $\mu$ at $I \in \mathcal{I}_{i}$ greater than the actual expected utility value at $I$. If he is rational at any $I \in \mathcal{I}_{i}$, then $i$ is said to be $\mu$-rational. Formally, let $\mathcal{R}_{i}^{\mu}(I)$ denote the event:

$$
\left.R_{i}^{\mu}(I):=\bigcap_{b_{i}^{\prime} \in \mathcal{B}_{i}} \sim K_{i}\left[U_{i}^{\mu}\left(b_{i}^{\prime}, \mathbf{b}_{-i}\right) \mid I\right) \supsetneqq U_{i}^{\mu}(\mathbf{b} \mid I)\right],
$$

where $\sim$ denotes the complementation and $\mathbf{b}_{-i}$ denotes $\left(\mathbf{b}_{j}\right)_{j \in N \backslash\{i\}}$. This is interpreted as the event that 'player $i$ is $\mu$-rational at $I \in \mathcal{I}_{i}$.' We denote

$$
R_{i}^{\mu}=\bigcap_{I \in \mathcal{I}_{i}} R_{i}^{\mu}(I) \quad \text { and } \quad R^{\mu}=\bigcap_{i \in N} R_{i}^{\mu} .
$$

The former event is interpreted as that player $i$ is $\mu$-rational and the latter as that all players are $\mu$-rational. Furthermore we define the notion of $\mu$-consistency. For given $\mu \in \mathcal{M}$, the event of $\mu$-consistency $C^{\mu}$ is the set of all the states $\omega$ such that there exists a sequence $\left\{\left(b^{n}, \mu\left(\cdot \mid b^{n}\right)\right)\right\} \subseteq \mathcal{B}^{+} \times \mathcal{M}^{+}$with $\lim _{n \rightarrow \infty}\left(b^{n}, \mu\left(\cdot \mid b^{n}\right)\right)=(\mathbf{b}(\omega), \mu)$.

It is well end this section in a remark: Rationality in perfect information game is clearly equivalent to $\mu$-rationality when the belief $\mu$ is the constant function 1 . That is, the rationality in Aumann (1995) is the 1-rationality $R^{1}$ for all players in our sense. One of the purposes in this paper is to extend the result of Aumann (1995) in the case of $\mu$-rationality. 


\section{The Result}

Let $G$ be an extensive form game and $\mu \in \mathcal{M}$. We denote by $S E^{\mu}(G)$ the event consisting of the states $\omega \in \Omega$ such that the assessment $(\mathbf{b}(\omega), \mu) \in \mathcal{B} \times \mathcal{M}$ constitutes a sequential equilibriums in $G$; that is,

$$
S E^{\mu}(G)=\{\omega \in \Omega \mid(\mathbf{b}(\omega), \mu) \in \mathcal{S E}(G)\} .
$$

Furthermore, $S E^{\mu}(G \mid I)$ denotes the event consisting of the states $\omega \in \Omega$ such that $(\mathbf{b}(\omega), \mu)$ is a member of $\mathcal{S E}(G \mid I)$ for each information set $I$.

We denote by $\mathcal{I}_{F}$ the subset of $\mathcal{I}$ consisting of all the information sets in which each player finally decides in the game $G$. By final decisions of player $i$ we mean the set of all the nodes in his information sets where he can reach some terminal node without any other his decisions. Let $R_{F}^{\mu}$ be the event of $\mu$-rationality over $\mathcal{I}_{F}$, that is, $R_{F}^{\mu}=\cap_{h \in \mathcal{I}_{F}} R_{i}^{\mu}(h)$.

The main theorem states that if $\mu$-rationality at the information sets in final decisions for each players under $\mu$-consistency for some $\mu \in \mathcal{M}$ is mutually known then the sequential equilibrium is achieved in the given game $G$. We can now state the main theorem formally as follows: Notations and assumptions being the same as above,

Theorem 3.1. $K_{E}\left(R_{F}^{\mu} \cap C^{\mu}\right)=S E^{\mu}(G)$.

Before proceeding with the proof we shall give two examples to illustrate the theorem.

Example 2. The first example is an extensive game with perfect information. $N$ is $\{1,2\}$. First player 1 chooses either $L$ or $R$ as his action, and player 2 chooses a pair of $a, b, c, d$ contingently. The backward induction strategy is uniquely determined: the player 1's is $R$ and the player 2's is $(b, d)$. Figure 1 shows this situation.

Now let $\left\langle\Omega,\left(\Pi_{i}\right)_{i \in N},\left(K_{i}\right)_{i \in N}, \mathbf{b}\right\rangle$ be a knowledge structure on $G$ consisting of as 


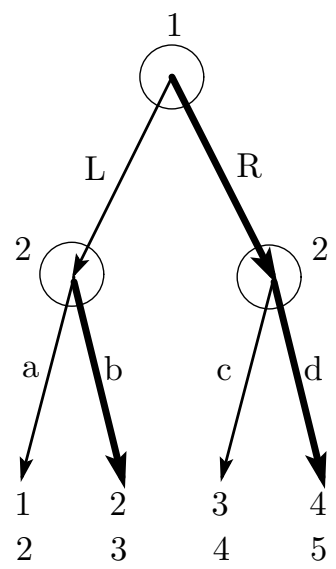

Figure 1: Case of Perfect Information

follows: $\Omega$ is the set of all action profiles:

$$
\Omega=\left\{\begin{array}{ll}
(L,(a, c)), & (R,(a, c)) \\
(L,(a, d)), & (R,(a, d)) \\
(L,(b, c)), & (R,(b, c)) \\
(L,(b, d)), & (R,(b, d))
\end{array}\right\}
$$

the knowledge partition $\Pi_{i}$ for $i \in N$ is defined by $\Pi_{i}(\omega)=\{\omega\}$ for every $\omega \in \Omega$; the knowledge operator $K_{i}$ is given by (2.6); and finally the map $\mathbf{b}: \Omega \rightarrow \mathcal{B}$ is the identity map. It is easily seen that $S E^{1}(G)=\{(R,(b, d))\}$ because the sequential equilibrium implies the backward induction strategy, and it can be also observed that $R^{1} \cap C^{1}=\{(R,(b, d))\}$. Therefore $K_{E}\left(R^{1} \cap C^{1}\right)=S E^{1}(G)$.

Example 3. The next example is an extensive game with imperfect information. $N$ is $\{1,2\}$. First player 1 chooses either $L$ or $R$ and player 2 chooses either $a$ or $b$. The sequential equilibrium uniquely determined: the player 1's is $R$ and the player 2 's is $b$ when $\mu(R)=1$. Figure 3 shows this situation.

Now let $\left\langle\Omega,\left(\Pi_{i}\right)_{i \in N},\left(K_{i}\right)_{i \in N}, \mathbf{b}\right\rangle$ be the knowledge structure on $G$ consisting of as follows: $\Omega$ is the set of all behavior strategies on $A$; that is, $\Omega=\mathcal{B}$. The knowledge partition $\Pi_{i}$ for player $i \in N$ is defined by $\Pi_{i}(\omega)=\{\omega\}$; the knowledge operator $K_{i}$ is given by (2.6); and finally the map $\mathbf{b}: \Omega \rightarrow \mathcal{B}$ is the identity map. 


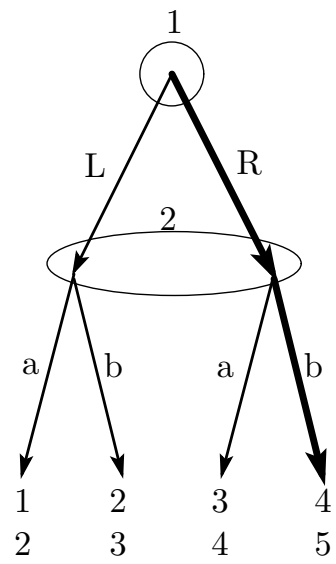

Figure 2: Case of Imperfect Information

It is easily seen that $S E^{\mu}(G)=\{(R, b)\}$ with $\mu(R)=1$ and it can be also observed that $R^{\mu} \cap C^{\mu}=\{R, b\}$. Therefore $K_{E}\left(R^{\mu} \cap C^{\mu}\right)=S E^{\mu}(G)$.

\section{Proof of Theorem}

On noting the assumption (PK) it can be plainly observed that $S E^{\mu}(G) \subseteq$ $K_{E}\left(R_{F}^{\mu} \cap C^{\mu}\right)$. The converse will be shown by induction as follows. It may be assumed that $K_{E}\left(R_{F}^{\mu} \cap C^{\mu}\right) \neq \emptyset$. For each information set $I \in \mathcal{I}_{i}$, let $S_{i}(I)$ be the subset of $\mathcal{I}_{i}$ consisting of $i$ 's information sets next after $i$ decides at $I$. Let $\mathcal{I}^{\prec}(I)$ denote the set of all the information sets at which $\iota(I)$ decides after $I$. We shall show the two points: First that for each $i \in N$ and any $h \in \mathcal{I}_{F} \cap \mathcal{I}_{i}, K_{i}\left(R_{F}^{\mu} \cap C^{\mu}\right) \subseteq$ $S E^{\mu}(G \mid h)$, and secondly that $K_{i}\left(R_{F}^{\mu} \cap C^{\mu}\right) \subseteq S E^{\mu}(G \mid I)$ if $K_{i}\left(R_{F}^{\mu} \cap C^{\mu}\right) \subseteq$ $S E^{\mu}(G \mid h)$ at any $h \in \mathcal{I}^{\prec}(I)$.

We shall verify the first point: For each player $i \in N$, it follows that

$$
K_{E}\left(R_{F}^{\mu} \cap C^{\mu}\right) \subseteq \bigcap_{b_{i}^{\prime} \in \mathcal{B}_{i}} \sim K_{i}\left[U_{i}^{\mu}\left(b_{i}^{\prime}, \mathbf{b}_{-i} \mid h\right) \supsetneqq U_{i}^{\mu}(\mathbf{b} \mid h)\right] \cap C^{\mu} .
$$

We note that for any $\omega \in K_{E}\left(R_{F}^{\mu} \cap C^{\mu}\right)$ and for any $b_{i}^{\prime} \in \mathcal{B}_{i}$,

$$
\begin{aligned}
\omega & \notin \quad K_{i}\left[U_{i}^{\mu}\left(b_{i}^{\prime}, \mathbf{b}_{-i} \mid I\right) \supsetneqq U_{i}^{\mu}(\mathbf{b} \mid I)\right] \\
& \Leftrightarrow \quad \exists \xi \in \Pi_{i}(\omega), \xi \notin\left[U_{i}^{\mu}\left(b_{i}^{\prime}, \mathbf{b}_{-i} \mid I\right) \supsetneqq U_{i}^{\mu}(\mathbf{b} \mid I)\right] \\
& \Leftrightarrow \quad \exists \xi \in \Pi_{i}(\omega), U_{i}^{\mu}(\mathbf{b}(\xi) \mid I) \geqq U_{i}^{\mu}\left(b_{i}^{\prime}, \mathbf{b}_{-i}(\xi) \mid I\right) .
\end{aligned}
$$


Furthermore, it is observed that $\mathbf{b}_{i}(\omega)=b_{i}(\xi)$ for any $\xi \in \Pi_{i}(\omega)$ by $(\mathbf{P K})$, and thus it can be plainly obtained that for any $\omega \in K_{E}\left(R_{F}^{\mu} \cap C^{\mu}\right)$ and for any $b_{i}^{\prime} \in \mathcal{B}_{i}$,

$$
U_{i}^{\mu}(\mathbf{b}(\omega) \mid I) \geqq U_{i}^{\mu}\left(b_{i}^{\prime}, \mathbf{b}_{-i}(\omega) \mid I\right) .
$$

Therefore we have shown that for each $\omega \in K_{i}\left(R_{F}^{\mu} \cap C^{\mu}\right)$, the assessment $(\mathbf{b}(\omega), \mu)$ is $\mu$-rational on any $h \in \mathcal{I}_{F} \cap \mathcal{I}_{i}$, and it is easily observed that the assessment is $\mu$-consistent. It follows that $K_{i}\left(R_{F}^{\mu} \cap C^{\mu}\right) \subseteq S E^{\mu}(G \mid h)$.

The following lemma is needed to verify the second point. For $I \in \mathcal{I}_{i}$ let $S_{i}(I)$ be the set of all nodes $x$ in $X_{i}$ such that there is a node $y$ in $I \cap P(x)$ with the property: If $z \in X_{i} \cap P(x)$ with $y \prec z \prec x$ then $z=y$. We denote $\boldsymbol{P}(x \mid b):=\boldsymbol{P}^{b}(x)$ for simplicity.

Lemma 4.1. For $b \in \mathcal{B}$, each $i \in N$, and for $I \in \mathcal{I}_{i}$ such that $S_{i}(I) \neq \emptyset$,

$$
U_{i}^{\mu}(b \mid I)=\sum_{h \in S_{i}(I)} \frac{\sum_{\tilde{x} \in h} \boldsymbol{P}(\tilde{x} \mid b)}{\sum_{\hat{x} \in h} \boldsymbol{P}(\hat{x} \mid b)} U_{i}^{\mu}(b \mid h) .
$$

Proof. For $b \in \mathcal{B}, x \in I$ and $x^{\prime} \in h \in S_{i}(I)$, it can be observed that

$$
\mu\left(x \mid b^{n}\right)=\frac{\sum_{\tilde{x} \in h} \boldsymbol{P}\left(\tilde{x} \mid b^{n}\right) \mu\left(x^{\prime} \mid b^{n}\right)}{\sum_{\hat{x} \in h} \boldsymbol{P}\left(\hat{x} \mid b^{n}\right) \prod_{a \in \pi\left(x, x^{\prime}\right)} b^{n}(a)} .
$$

Therefore it follows that

$$
\begin{aligned}
U_{i}^{\mu}(b \mid I) & =\lim _{n \rightarrow \infty} \sum_{x \in I} \mu\left(x \mid b^{n}\right) \prod_{a \in \pi(x, z)} b^{n}(a) u_{i}(z) \\
& =\lim _{n \rightarrow \infty} \sum_{x \in I} \frac{\sum_{\tilde{x} \in h} \boldsymbol{P}\left(\tilde{x} \mid b^{n}\right)}{\sum_{\hat{x} \in I} \boldsymbol{P}\left(\hat{x} \mid b^{n}\right)} \mu\left(x^{\prime} \mid b^{n}\right) \prod_{a \in \pi\left(x^{\prime}, z\right)} b(a) u_{i}(z) \\
& =\lim _{n \rightarrow \infty} \sum_{h \in S_{i}(I)} \frac{\sum_{\tilde{x} \in \mathrm{h}} \boldsymbol{P}\left(\tilde{x} \mid b^{n}\right)}{\sum_{\hat{x} \in I} \boldsymbol{P}\left(\hat{x} \mid b^{n}\right)} \sum_{x^{\prime} \in h} \mu\left(x^{\prime} \mid b^{n}\right) \prod_{a \in \pi\left(x^{\prime}, z\right)} b(a) u_{i}(z) \\
& =\sum_{h \in S_{i}(I)} \frac{\sum_{\tilde{x} \in \mathrm{h}} \boldsymbol{P}(\tilde{x} \mid b)}{\sum_{\hat{x} \in I} \boldsymbol{P}(\hat{x} \mid b)} U_{i}^{\mu}(b \mid h),
\end{aligned}
$$

in completing the proof of the lemma.

Let us return to the proof of theorem, and we shall verify the second point. Assume now that $K_{i}\left(R_{F}^{\mu} \cap C^{\mu}\right) \subseteq S E^{\mu}(G \mid h)$ for each $i \in N$ and each information 
set $h \in \mathcal{I}_{i}$ with $h \subseteq S_{i}(I)$. We shall show that $K_{i}\left(R_{F}^{\mu} \cap C^{\mu}\right) \subseteq S E^{\mu}(G \mid I)$. Suppose to the contrary that there exists $\tilde{b}_{i} \in \mathcal{B}_{i}$ such that at $\omega \in K_{i}\left(R_{F}^{\mu} \cap C^{\mu}\right)$,

$$
U_{i}^{\mu}\left(\tilde{b}_{i}, \mathbf{b}_{-i}(\omega) \mid I\right) \supsetneqq U_{i}^{\mu}(\mathbf{b}(\omega) \mid I) .
$$

It suffices to prove that for any $\bar{b}_{i} \in \mathcal{B}_{i}$

$$
U_{i}^{\mu}(\mathbf{b}(\omega) \mid I) \geqq U_{i}^{\mu}\left(\bar{b}_{i}, \mathbf{b}_{-i}(\omega) \mid I\right) .
$$

In fact, it can be easily observed that the inequality (4.12) is in contradiction to (4.11), completing the proof of the theorem.

We shall verify the inequality (4.12) as follows: It follows from the above lemma that

$$
\begin{aligned}
U_{i}^{\mu}(\mathbf{b}(\omega) \mid I)= & \sum_{h \in I} \frac{\sum_{\tilde{x} \in h} \boldsymbol{P}(\tilde{x} \mid \mathbf{b}(\omega))}{\sum_{\hat{x} \in I} \boldsymbol{P}(\hat{x} \mid \mathbf{b}(\omega))} U_{i}^{\mu}(\mathbf{b}(\omega) \mid h) \\
\geqq & \sum_{h \in S_{i}(I) \backslash\left\{h^{\prime}\right\}} \frac{\sum_{\tilde{x} \in h} \boldsymbol{P}(\tilde{x} \mid \mathbf{b}(\omega))}{\sum_{\hat{x} \in I} \boldsymbol{P}(\hat{x} \mid \mathbf{b}(\omega))} U_{i}^{\mu}(\mathbf{b}(\omega) \mid h) \\
& +\frac{\sum_{\tilde{x} \in h} \boldsymbol{P}(\tilde{x} \mid \mathbf{b}(\omega))}{\sum_{\hat{x} \in I} \boldsymbol{P}(\hat{x} \mid \mathbf{b}(\omega))} U_{i}^{\mu}\left(\bar{b}_{i}, \mathbf{b}_{-i}(\omega) \mid h^{\prime}\right) \\
= & \lim _{n \rightarrow \infty} \sum_{h \in S_{i}(I) \backslash\left\{h^{\prime}\right\}} \frac{\sum_{\tilde{x} \in h} \boldsymbol{P}\left(\tilde{x} \mid b^{n}\right)}{\sum_{\hat{x} \in I} \boldsymbol{P}\left(\hat{x} \mid b^{n}\right)} U_{i}^{\mu\left(\cdot \mid b^{n}\right)}\left(b^{n} \mid h\right) \\
& +\lim _{n \rightarrow \infty} \frac{\sum_{\tilde{x} \in h} \boldsymbol{P}\left(x^{\prime} \mid b^{n}\right)}{\sum_{\hat{x} \in I} \boldsymbol{P}\left(\hat{x} \mid b^{n}\right)} U_{i}^{\mu\left(\cdot \mid b^{n}\right)}\left(\bar{b}_{i}^{n}, b_{-i}^{n} \mid h^{\prime}\right) \\
= & U_{i}^{\mu}\left(\bar{b}_{i}, \mathbf{b}_{-i}(\omega) \mid I\right) .
\end{aligned}
$$

This completes the proof of the inequality (4.12) and so does the proof of the theorem.

\section{Concluding Remarks}

This paper examines what epistemic conditions about players' rationality lead to the outcomes induced by a sequential equilibrium. Aumann (1995) shows that if players act on the rational behavior in a perfect information game then they can obtain the outcome by the backward induction solution. In this paper we extend this result into the case for sequential equilibrium. We require here only the mutual knowledge of rationality for all players instead of common knowledge of it in Aumann (1995). 
Therefore it is sufficient only to know rationality at the information sets in final decisions for each player.

Some related works (e.g. Reny (1992), Ben-Porath (1997)) lead to the different results from Aumann's. In Aumann (1995) and this paper rationality on information sets is required, however they do only beliefs about players' rationality at the beginning of a game. Since players have the Bayesian rationality in Reny (1992), Ben-Porath (1997) players can revise their own beliefs about their opponents' behaviors or their present nodes through moving plays. These are the different views

in examining extensive form games. Aumann regards rationality of players as an representation of the equilibrium, while Reny and Ben-Porath capture it as playability in a given game. We would like to examine the relationship between the two views in the further research.

\section{References}

Aumann, R. J. (1995). "Backward induction and common knowledge of rationality". Games and Economic Behavior, Vol. 8, 6-19.

Aumann, R. J. and A. Brandenburger (1995). "Epistemic conditions for Nash equilibrium". Econometrica, Vol. 63, No. 5, 1161-1180.

Bacharach, M. (1985). "Some extensions of a claim of Aumann in an axiomatic model of knowledge". Journal of Economic Theory, Vol. 37, 167-190.

Ben-Porath, E. (1997). "Rationality, Nash equilibrium and backwards induction in perfectinformation games". Review of Economic Studies, Vol. 64, 23-46.

Kreps, D. M. and R. Wilson (1982). "Sequential equilibria". Econometrica, Vol. 50, No. 4, 863-894.

Kuhn, H. W. (1953). "Extensive games and the problem of information". In H. W. Kuhn and A. W. Tucker (eds.). Contributions to the Theory of Games II, Annals of Mathematical Studies. Princeton: Princeton University Press, No. 28, 193-216,

Reny, P. J. (1993). "Common belief and the theory of games with perfect information". Journal of Economic Theory, Vol. 59, 257-274.

Rosenthal, R. W. (1981). "Games of perfect information, predatory pricing and the chainstore paradox". Journal of Economic Theory, Vol. 25, 92-100. 